\title{
Generalized Breadths, Circular Cantor Sets, and the Least Area UCC
}

\author{
Gy. Elekes \\ Eötvös University, Múzeum krt. 6-8, \\ H-1088 Budapest, Hungary \\ elekes@cs.elte.hu
}

Communicated by Imre Bárány

Dedicated to the memory of Zs. Baranyai

\begin{abstract}
We develop a technique suitable for determining the minimal area convex set that can cover certain prescribed regular polygons. As a side effect we improve the well-known "circle-and-triangle" lower bound on the least area Universal Convex Cover (UCC).
\end{abstract}

\section{Introduction}

An oval is a compact, convex set with a nonempty interior in the euclidean plane. The diameter of an oval is the longest distance that occurs between two of its points. A Universal Convex Cover (UCC for short) is an oval which, if shifted and rotated appropriately, can cover any given oval of unit diameter. (Note that arbitrary nonconvex sets of unit diameter can also be covered by these UCCs since taking closures and/or convex hulls does not increase the diameter.)

It was observed by Jung [J2] that a disk of radius $1 / \sqrt{3}$ is a UCC. The following question was formulated by Lebesgue in 1914 (see [P1]):

Determine the least area Universal Convex Cover.

Standard compactness arguments show that the minimum does indeed exist.

A UCC smaller than Jung's disk was discovered by Pál [P1], [P2] who demonstrated this feature of a regular hexagon of unit breadth. He also observed that two corners of this hexagon can be truncated without spoiling the UCC 


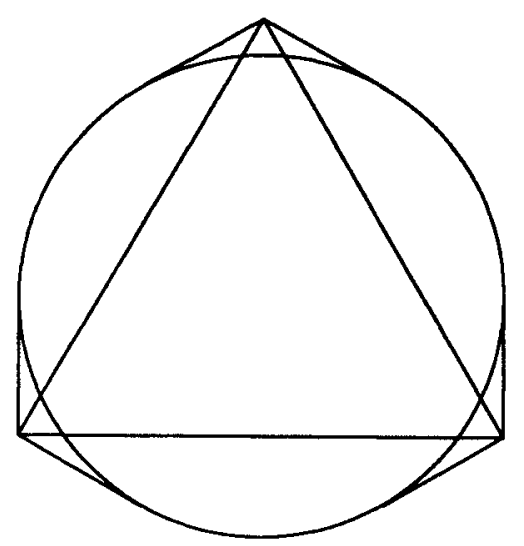

Fig. 1. The "circle-and-triangle" configuration.

property. Since then the problem has been studied by several people, e.g., Sprague [S], Hansen [H1], [H2], and Duff [D], whose results all go in one direction: they chop off more and more of Pál's hexagon while maintaining the UCC property. (Related questions like covering triangles or covering by translates are considered by, e.g., Kovalev [K], Wetzel [W1]-[W3], Nitche [N], and Bezdek and Connelly [BC].)

Little is known in the other direction, ie., at least how large the area of a UCC should be. Of course it cannot be smaller than the minimal area oval that contains a disk of unit diameter as well as an equilateral triangle of this side length (see Fig. 1). This "circle-and-triangle" configuration looks like Pál's hexagon with three corners rounded off and contains a good proportion of the area of the latter (let alone that of the above mentioned truncated UCCs). It may be felt that this oval is a good candidate for a least area UCC.

However, this is not the case. It is mentioned (without proof) in [P1] that the circle-and-triangle is insufficient for covering, e.g., a regular pentagon (of unit diameter, of course). This has recently been rediscovered by Tóth [T] but even he was unable to improve the lower bound for the area of a UCC.

It is natural to ask whether it is easier to determine minimal covers for just a few ovals (like the above disk and triangle). The aim of this paper is to study certain such "restricted convex covers" (CC for short) which will be defined to be suitable for covering some, but not all, sets of unit diameter. The ovals to be covered will usually be regular polygons and a disk. We determine the minimum circumference as well as the minimum area of certain such CCs. As a side effect this provides lower bounds for the UCC also.

(For those who prefer figures: the area of the circle-and-triangle lower bound is 0.8257 while the best upper bound (due to Hansen) is 0.8441 . Our results, if applied to the case of all regular $3^{j}$-gons $(j \geq 1)$, give 0.8271 which fills up $7 \%$ of the gap between the above-mentioned estimates, leaving abundant space for further development.) 
The CCs we construct are all alike; they come from certain "Cantor-type" patterns (see the next section). The main tools for proving that our covers are really minimal is what we call " $s$-extents" which are generalizations of the usual notion of breadth.

\section{Notation and Definitions}

Throughout this paper $d(K), a(K)$, and $c(K)$ denote the diameter, area, and circumference, respectively, of an oval $K$. We put $R_{n}$ for a regular $n$-gon of unit diameter and $D$ for a disk, also of this diameter. (Note that, for even values of $n$, $R_{n}$ is inscribed into $D$ which is not the case for odd $n$ 's; regular star-shaped polygons of unit edge-length do not fit into $D$.)

Restricted $C$ Cs. Let $\mathscr{P}$ be an arbitrary set of ovals. We define

$$
\mathrm{CC}(\mathscr{P}):=\{K \text { oval; } K \text { can cover every } P \in \mathscr{P}\} \text {. }
$$

Moreover, for the least area/circumference a $\mathrm{CC}$ for $\mathscr{P}$ can have, we put

$$
\begin{aligned}
& a(\mathscr{P}):=\min \{a(K) ; K \in \mathrm{CC}(\mathscr{P})\}, \\
& c(\mathscr{P}):=\min \{c(K) ; K \in \mathrm{CC}(\mathscr{P})\} .
\end{aligned}
$$

We hope that using $a$ and $c$ for two, apparently different, purposes will cause no confusion since the minimal area/circumference oval that contains a given oval $K$ is $K$ itself, ie., $a(\{K\})=a(K)$ and, similarly, $c(\{K\})=c(K)$.

Using the above notations

$$
a(\{P ; d(P)=1\})
$$

is the quantity Lebesgue wanted to know.

More Special CCs. In what follows the set $\mathscr{P}$ of ovals usually consists of some regular polygons $R_{n}$ together with, possibly, a disk $D$-all with unit diameter. That is why we introduce another shorthand (actually two).

Definition 1. For $I \subset\{3,4,5, \ldots\}$ we write

$$
\mathrm{CC}(I):=\mathrm{CC}\left(\left\{R_{i} ; i \in I\right\}\right)
$$

and, similarly,

$$
\mathrm{CC}(\{D\} \cup I):=\mathrm{CC}\left(\{\mathrm{D}\} \cup\left\{\mathrm{R}_{i} ; i \in I\right\}\right)
$$

Remark. Of course this last notion is interesting only if $I$ consists of odd integers; otherwise all the $R_{2 n}$ can be covered just by $D$. 
We also extend the usage of $a$ and $c$ even further and put

$$
\begin{gathered}
a(I):=\min \{a(K) ; K \in \mathrm{CC}(I)\}, \\
c(I):=\min \{c(K) ; K \in \mathrm{CC}(I)\}, \\
a(\{D\} \cup I):=\min \{a(K) ; K \in \mathrm{CC}(\{D\} \cup I)\}, \\
c(\{D\} \cup I):=\min \{c(K) ; K \in \mathrm{CC}(\{D\} \cup I)\},
\end{gathered}
$$

which, again, might cause some ambiguities, but we cross our fingers.

\section{Cantor-Type Arrangements}

Let $n \geq 3$ be an odd integer. Denote the center of the circle circumscribed around $R_{n}$ by $\mathrm{O}$ and call it the center of $R_{n}$ for short.

Place disk $D$ of unit diameter on $R_{n}$ in such a way that its center coincides with o. Draw two tangent lines from each vertex $v$ of $R_{n}$ to $D$ and denote the (shorter) arc of the boundary of $D$ between these lines by $\overline{\mathbf{v}}$. Moreover, call the region bounded by $\overline{\mathbf{v}}$ and the corresponding segments of the tangent lines the cap determined by $\mathbf{v}$. We denote it by $\hat{\mathbf{v}}$. Sometimes we say that $\overline{\mathbf{v}}$ is the base arc of $\hat{\mathbf{v}}$ or, equivalently, that $\hat{\mathbf{v}}$ covers $\overrightarrow{\mathbf{v}}$ (see Fig. 2).

Proposition 2. The length of the arcs $\overline{\mathbf{v}}$ as well as of those which are not covered by the caps are all equal to $\pi / 2 n$.

We want to place $D$ and two regular polygons, say $R_{n}$ and $R_{m}$ with $n<m$, on each other in such a way that the "big arcs" (determined by the vertices of $R_{n}$ ) cover as much of the "small arcs" (that correspond to the vertices of $R_{m}$ ) as possible.

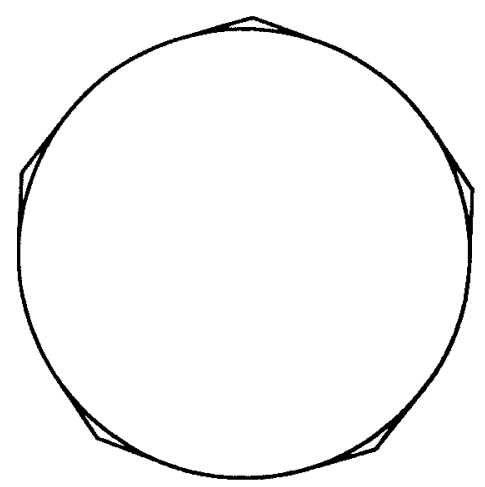

Fig. 2. Caps of $D$ formed by $R_{5}$. 
Definition 3. Let $n$ and $m$ be odd integers for which $n \mid m$. An arrangement of $D$, $R_{n}$, and $R_{m}$ is called canonical if:

(1) Their centers coincide.

(2) Each of the "big arcs" $\overline{\mathbf{v}}\left(\mathbf{v} \in R_{n}\right)$ is divided into equal parts by the "small $\operatorname{arcs} " \overline{\mathbf{w}}$ (for $\mathbf{w} \in R_{m}$ ).

(3) All the arcs $\bar{v}$ for $v \in R_{n}$ start and end with arcs $\bar{w}$ for $w \in R_{m}$. Consequently, the "big arcs" not covered by "big caps" start and end with "small arcs" not covered by "small caps."

(Such a placement of the objects is made possible by the parity and divisibility assumptions on $n$ and $m$.)

Now we describe our candidates for the least area/circumference $\operatorname{CC}(\{D\} \cup I)$ of certain sets $I$ of the integers.

Definition 4. Let $I$ be a set of odd integers at least 3. Assume that $n, m \in I$ and $n<m$ implies $n \mid m$. We call a placement of $D$ and the regular polygons $\left\{R_{n} ; n \in I\right\}$ a canonical pattern or a Cantor-type arrangement if it is canonical for each pair $n, m$ in $I$.

Remark. Actually, in what follows, we prefer the expression "Cantor-type." The reason for this is that it visualizes an interesting property of such configurations: the union of the base-arcs $\overline{\mathbf{v}}$, while $n$ ranges over $I$ and $\mathbf{v}$ over the vertices of $R_{n}$, produces a pattern that is quite similar to the intervals used in the definition of Cantor's set in $[0,1]$. If, for example, $I$ is the set of the powers of 3 , then the system of these arcs can be considered as the circular re-incarnation of that well-known set.

\section{Results and Open Problems}

Our main result is the following:

Theorem 1. Let I be a (finite or infinite) set of odd integers at least 3. Assume that $n, m \in I$ and $n<m$ implies $n \mid m$. Then both $c(\{D\} \cup I)$ and $a(\{D\} \cup I)$ are attained for the convex hull of the corresponding Cantor-type arrangement.

Each such configuration with $\{3\} \subseteq I$ is strictly bigger than the "disk-andtriangle" of Fig. 1. Hence each of them gives an improvement on the lower bound for the least area UCC. The best of the Cantor-type arrangements, for any set $I$ of odd integers, is the case of the powers of 3 which yields the following assertion. 
Corollary 5. The circumference of a UCC is at least

$$
3\left[\tan \left(\frac{\pi}{6}\right)+\sum_{i=0}^{\infty} 2^{i} \tan \left(\frac{\pi}{18 \cdot 3^{i}}\right)\right],
$$

while its area is at least $\frac{1}{4}$ times this quantity.

(Both of these estimates come from the Cantor-type arrangement of the $3^{i}$-gons for all $i \geq 1$.)

Several problems (beyond Lebesgue's original question) remain open.

The reader may have observed that $a(I)$ and $c(I)$ (without $D$ ) do not occur in Theorem 1. It can be shown that the assertion holds for $c(I)$ as well, by following the steps of the first part of the proof of the theorem and modifying them appropriately. However, unlike to $a(\{D\} \cup I)$, the Simultaneous Minimum Lemma cannot be applied to $a(I)$. Does the statement of Theorem 1 hold true for this quantity, too? We do not know the answer.

The original UCC problem seems much harder to settle. To justify this feeling, it is worth noting that in the configuration that attains $a(\{D\} \cup\{3,5\})$, the centers do not coincide. This motivates the formulation of a general question.

Problem. Characterize those sets $I$ for which $a(\{D\} \cup I)$ is attained for a concentric arrangement of $D$ and the $R_{i}$.

The following special case seems plausible but we have not been able to prove it.

Conjecture. $I=\{3 n ; n \geq 1\}$ has this property. (Perhaps even the coefficient 3 can be replaced here by any bigger odd integer.)

The simplest case of the general problem where we cannot even decide what to guess is that of $I=\{3,5,15\}$. However, the same statement for the least circumference cover is not difficult to prove.

Theorem 2. If $s \geq 3$ is odd and $I=\{k s ; k \in \mathbf{N}\}$, then among the arrangements of $\left\{R_{i} ; i \in I\right\}$ (resp. among those of $\{D\} \cup\left\{R_{i} ; i \in I\right\}$ ) that attain the minimum circumference $c(I)$ (resp. $c(\{D\} \cup I)$ ) there is one whose $R_{i}$ 's (together with $D$ ) are all concentric.

\section{Cantor-Type Arrangements and the "Cog-Wheel Lemma"}

From our point of view, the importance of these configurations is that, for any finite set $I$, no other concentric pattern can leave so much of the boundary of $D$ uncovered. The goal of this section is to formulate this assertion exactly. 
Lemma 6 ("Cog-Wheel Lemma"). Let $n_{1}<n_{2}<\cdots<n_{i}<\cdots$ be a finite or infinite sequence of odd integers. Assume that $n_{i} \geq 3$ and $n_{i} \mid n_{i+1}$ for all possible values of $i$. For each $i$ cut the circumference of a given circle $C$ into $2 n_{i}$ equal arcs arbitrarily and take every other one of them to form a set $S_{i}$ of $n_{i}$ arcs for each $i$. (They resemble cog-wheels which gives the name of the lemma.) Then it is possible to pick a subset $S_{i}^{\prime}$ of the arcs in $S_{i}$ for each $i$ such that:

(1) The arcs in $S_{i}^{\prime}$ and $S_{j}^{\prime}$ have no inner point in common for $i \neq j$.

(2) $S_{i}^{\prime}$ consists of exactly that many arcs as the number of "smallest caps" not covered by "bigger caps" in the Cantor-type arrangement of $D$ and the $n_{j}$-gons for $j \leq i$.

Proof. Put $q:=n_{i} / n_{j}$ for some pair $j<i$. ( $q$ is an odd integer.) Then the common length of the arcs in $S_{j}$ is $q$ times those in $S_{i}$. Since the latter alternate with "empty arcs" of the same length, no arc of $S_{j}$ can intersect more than $\lceil q / 2\rceil=(q+1) / 2$ of them; equality holds, among others, for Cantor-type arrangements. Now you can proceed by the greedy method of selecting $S_{i}$ inductively.

Remark. The exact number of arcs in $S_{i}^{\prime}$ is of no use to us; only the property stated above is important. That is why we avoided computing these figures.

\section{s-Extents as Generalized Breadths}

This section describes our main tools for proving the minimality of certain CCs.

Let $K$ be an arbitrary oval and $\mathbf{p} \in K$. For any angle $\alpha \in[0,2 \pi)$ denote by $h^{\mathbf{p}, K}(\alpha)$ the support function of $K$ with respect to $p$, i.e., the distance of $\mathbf{p}$ from the support line $l(\alpha)$ of $K$ (see Fig. 3).

Definition 7. Let $K$ and $\mathbf{p}$ be as above. Moreover, let $s \geq 1$ be an arbitrary integer. For $\alpha \in[0,2 \pi)$ put

$$
e_{s}^{\mathrm{p}, K}(\alpha):=\sum_{m=0}^{s-1} h^{\mathrm{p}, K}\left(\alpha+m \frac{2 \pi}{s}\right)
$$

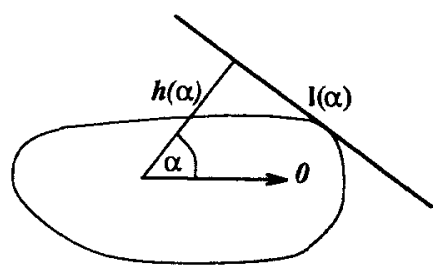

Fig. 3. Support line and $h$ in direction $\alpha$. 
We call the above quantity the s-extent of $K$ in directions

$$
\alpha, \alpha+2 \pi / s, \ldots, \alpha+(s-1)(2 \pi / s)
$$

Remark. $e_{1}$ is just $h$ defined above while $e_{2}$ is the usual notion of breadth, independently from the choice of $\mathbf{p}$. This last observation can be further generalized.

Lemma 8. For $s \geq 2, e_{s}^{\mathrm{p}, K}(\alpha)$ is independent of $\mathbf{p}$.

Proof. Consider the convex polygon formed by the $s$ support lines and use the well-known fact that if all angles of an $s$-gon are $(1-2 / s) \pi$, then the sum of the distances of an inner point from the lines that contain the edges is constant (i.e., does not depend on the choice of the point).

That is why, in what follows, we may just write $e_{s}^{K}(\alpha)$ or, if $K$ is obvious, simply $e_{s}(\alpha)$ instead of $e_{s}^{\mathrm{p}, K}(\alpha)$.

We generalize a well-known relation between circumference and breadth for $s$-extents. Denote by $b^{K}(\alpha)$ the breadth of an oval $K$ in direction $\alpha$ and, as usual, by $c(K)$ the circumference of $K$. The following formula of Cauchy can be found, e.g., in $[\mathrm{BF}]$ :

$$
\int_{0}^{2 \pi} b^{K}(\alpha) d \alpha=2 \cdot c(K)
$$

Lemma 9 (Basic Lemma). For an arbitrary oval $K$ and natural number $s \geq 1$,

$$
\int_{0}^{2 \pi} e_{s}^{K}(\alpha) d \alpha=s \cdot c(K) .
$$

Proof. True for $s=2$ by $b(\alpha)=e_{2}(\alpha)$ and (*). The case $s=1$ then comes from $e_{2}(\alpha)=e_{1}(\alpha)+e_{1}(\alpha+\pi)$ whence the general statement follows immediately.

\section{Least Area and Shortest Circumference}

In this section we show that, for certain classes $\mathscr{P}$ of ovals, the shortest circumference $\mathrm{CC}$ has the least area as well. The key observation is the fact that if a polygon $P$ has an inscribed circle, then the area of $P$ can be computed from its circumference and the radius of the circle.

First we extend the notion of an inscribed circle to nonpolygonal ovals.

Definition 10. We say that an oval $K$ possesses an inscribed circle $C$ (or, equivalently, $K$ is circumscribed around $C$ ) if the boundary of $K$ consists of a 
subset of $C$ together with some line segments that are all tangents to $C$. (Bonnensen and Fenchel call these ovals "cap-bodies" in [BF].)

Proposition 11. If an oval $K$ contains a circle $C$ of radius $r$, then

$$
a(K) \geq \frac{r}{2} c(K)
$$

and equality holds if and only if $K$ is circumscribed around $C$ in the above sense.

Proof. Denote by $\rho(s)$ the distance between the center of $C$ and the line spanned by the vector ds, at the value $s$ of the arc-length parameter. This is defined almost everywhere and we have $\rho(s) \geq r$ almost everywhere with equality almost everywhere iff $C$ is an inscribed circle of $K$. Then

$$
a(K)=\frac{1}{2} \int_{\mathrm{bd} K} \rho(s) d s \geq \frac{1}{2} r \int_{\mathrm{bd} K} d s=\frac{1}{2} r c(K)
$$

with equality if and only if $K$ is circumscribed around $C$.

Now we can formulate why it is sometimes possible to reduce the problem of the least area to that of the shortest circumference.

Lemma 12 (Simultaneous Minimum Lemma). Assume that $\mathscr{P}$ is a class of ovals and $K_{0}$ is another oval (not necessarily in $\mathscr{P}$ ) for which the extremum

$$
c\left(K_{0}\right)=c(\{D\} \cup \mathscr{P})
$$

is attained. If, moreover, $K_{0}$ has an inscribed circle of unit diameter, then

$$
a\left(K_{0}\right)=a(\{D\} \cup \mathscr{P})
$$

Proof. Immediate from the previous proposition with $r=\frac{1}{2}$ since all CCs for $\{D\} \cup \mathscr{P}$ contain $D$.

\section{Proof of the Theorems}

Proof of Theorem 1 . Let $I$ be as in the theorem and put $s:=\min I$. Consider an oval $K$ that attains $c(K)=c(\{D\} \cup I)$. According to the Basic Lemma,

$$
c(K)=\frac{1}{s} \int_{0}^{2 \pi} e_{s}^{K}(\alpha) d \alpha .
$$


As $e_{s}^{R_{i}}(\alpha)$ (unlike $h^{R_{i}}(\alpha)$ ) is insensitive to shifting the $R_{i}$, we can use the Cog-Wheel Lemma to prove that this integral cannot be less than the one that corresponds to the Cantor-type arrangement. This demonstrates that these arrangements have the shortest possible circumference among the CCs. Then the Simultaneous Minimum Lemma yields the same result for their area.

Proof of Theorem 2. Consider an arbitrary arrangement of $I$ (resp. $\{D\} \cup I$ ). It suffices to show that there is a concentric arrangement whose circumference does not exceed that of the original one. To show this, start the other way round, from a concentric arrangement.

Lemma 13. If $s \geq 3$ odd and $I=\{k s ; k \in \mathbf{N}\}$, then shifting (but not rotating) the polygons $R_{i}(i \in I)$ of a concentric arrangement does not decrease $e_{s}(\alpha)$.

Proof of the Lemma. For a concentric arrangement and any fixed $\alpha \in[0,2 \pi)$, the terms of

$$
e_{s}(\alpha)=\sum_{m=0}^{s-1} e\left(\alpha+m \frac{2 \pi}{s}\right)
$$

come from one and the same $R_{i}$ (or $\left.D\right) ; e_{s}(\alpha)$ of the shifted system cannot be less than that of just one of its members $R_{i}$. Hence the circumference, which is the integral of $e_{s}(\alpha)$, cannot be bigger for the original concentric system.

To prove Theorem 2, consider a minimal arrangement and, if necessary, shift its members so that they become concentric. By the above lemma, the new system cannot be worse than the one we started from.

\section{Acknowledgments}

The author wishes to thank E. Makai, Jr., for his suggestions and comments on the first version of the manuscript. Moreover, he cannot find any better way of expressing his thanks to his late friend and colleague Zs. Baranyai who was the first to tell him about the UCC problem and several other beauties of mathematics.

\section{References}

[BC] K. Bezdek and R. Connelly, Covering curves by translates of a convex set, Amer. Math. Monthly 96 (1989), 789-806.

[BF] T. Bonnensen and W. Fenchel, Theorie der konvexen Körper, Springer-Verlag, Berlin, 1974.

[D] G. F. D. Duff, A smaller universal cover for sets of unit diameter, C. R. Math. Rep. Acad. Sci. Canada 2(1) (1980), 37-42.

[H1] H. C. Hansen, A small universal cover of figures of unit diameter, Geom. Dedicata 4 (1975), $165-172$.

[H2] H. C. Hansen, Small universal covers of sets of unit diameter, Geom. Dedicata 42 (1992), $205-213$. 
[J1] H. W. E. Jung, Über die kleinste Kugel, die eine raumliche Figur einschliesst, Crelles J. reine angew. Math. 123 (1901), 241-257.

[J2] H. W. E. Jung, Über den kleinsten Kreis, der eine ebene Figur einschliesst, Crelles J. reine angew. Math. 137 (1910), 310-313.

[K] M. D. Kovalev, A minimal convex covering for triangles, Ukrain. Geom. Sb. 26 (1983), 63-68 (in Russian).

[M] H. Meschkowski, Ungelöste und unlösbare Probleme der Geometrie, Vieweg, Braunschweig, 1960.

[N] J. C. C. Nitche, The smallest sphere containing a rectifiable curve, Amer. Math. Monthly 78 (1971), 881-882.

[P1] J. Pál, Über ein elementares Variationsproblem, Danske Vidensk. Selsk. Math.-fys. Medd. III 2 (1920), 3-35.

[P2] J. Pál, Ein Minimalproblem für Ovale, Math. Ann. 83 (1921), 311-319.

[S] R. Sprague, Über ein elementares Variationsproblem, Mat. Tid. (1936), 96-99.

[T] G. Tóth, Private communication.

[W1] J. E. Wetzel, Triangular covers for closed curves of constant length, Elem. Math. 25(4) (1970), $78-81$.

[W2] J. E. Wetzel, On Moser's problem of accommodating closed curves in triangles, Elem. Math. 27(2) (1972), 35-36.

[W3] J. E. Wetzel, Sectorial covers for curves of constant length, Canad. Math. Bull. 16 (1973), $367-375$.

Received April 22, 1993. 